\title{
17beta estradiol ameliorates neuroinflammation driven neuropathic pain in chronic constriction injury - induced peripheral neuropathy in rats
}

\author{
Nafissa Telailia', Hacène Frih', Sylvain Fisson ${ }^{2,3,4}$ \\ ${ }^{1}$ Environmental Biomonitoring Laboratory, Department of Biology, Faculty of Sciences, \\ Badji Mokhtar University, Annaba, Algeria \\ 2INSERM, UMR_S951, Evry, France \\ ${ }^{3}$ Genethon, Evry, France \\ ${ }^{4}$ University of Evry Val d’Essonne, UMRS 951, Evry, France
}

\begin{abstract}
Background. Accumulating evidence illustrates that peripheral nerve injuries ac-tivate glial components of the peripheral and central cellular circuitry which contributes to neuroinflammation and neuropathic pain. Epidemiological surveys suggest that for women the prevalence of pain increases with age with more frequent pain than men. Recently, several studies have found that pain generation, transmission and modulation are under hormonal regulation. To this end, 17beta estradiol was used to investigate the modulatory actions on nociceptive thresholds in neuroinflammation driven neuropathic pain conditions.

Methods. Adult female Wistar rats were divided into four groups: Ovariectomised neuropathic untreated and neuropathic 17 beta estradiol treated groups. sham operated and intact control groups.

Results. In treated rats, the results validate an anti-inflammatory action of 17 beta estradiol against chronic constriction injury $(\mathrm{CCl})$ induced neuropathic pain by attenuat-ing complement $\mathrm{C} 3$ fractions and anti-neutrophil cytoplasmic auto-antibodies (ANCAs) intensity plasma level, an anti-nociceptive action by attenuating ipsilateral, con-trolateral allodynia and hyperalgesia developed in treated animals after chronic con-striction injury and at the last day of treatment by increasing the tail flick latency at the last day of treatment.

Conclusions. We conclude that 17 beta estradiol might exhibit beneficial effects on peripheral nerve injury consequences.
\end{abstract}

Keywords: bilateral ovariectomy, chronic constriction injury, allodynia, hyperalgesia, up and down order method, 17 beta estradiol therapy

\section{INTRODUCTION}

As nerves leave the spine, they will course through the body and are vulnerable to compression or entrapment anywhere along its course, after a nerve is injured in the periphery, a complex and finely regulated sequence of events commences to remove the damaged tissue and begin the reparative process. Injury resulting from a compressive force lead to local myelin damage (1) where there is growing evidence suggesting that damage to the myelin sheath is due to a disturbed Schwann cell response in conjunction with immune cell participation (2-4) which contributes to local inflammato- ry response that consists of a proinflammatory phase in which any pathogens are removed, damaged cells and debris cleared, and the local homeostasis restored. After this, there is a phase of resolution in which there is a local tissue repair and the potentially damaging effects of an excessive inflammatory response in both the peripheral and the central nervous system are dissipated which may contribute to the initiation and maintenance of persistent pain (5). The role of peripheral immune cells has been highlighted in the last few studies. Although not found in healthy nerves, neutrophils (or polymorphonuclear leukocytes) are found in high 
numbers close $(<2 \mathrm{~mm})$ to the site of injury and participate in the very early stages following peripheral nerve injury, peaking at $24 \mathrm{~h}(6,7)$. There is rapid infiltration and transient presence of neutrophils after the lesion (8). Therefore, peripheral immune cells significantly contribute to the inflammatory environment (9). Based on available evidence, the main elements involved in neuroinflammation are microglia and the infiltrating immune cells. There is increasing research on the association between complement activation and neuropathic pain (10) where an increased C3a was found to provoke the production of inflammatory mediators, which in turn further enhanced synthesis of the complement. Thus, a feedback loop is formed between activation of inflammatory cytokines and the complement system, leading to nerve-immune activation (11). Peripheral nerve injuries lead to spontanoeus pain followed by allodynia and hyperalgesia (12, 13). Allodynia is a pain elicited by normally non painfully stimuli (14), it was quantified by Von Frey filaments, a highly sensitive test in detecting allodynia in conditions likely to cause neuropathic pain (15), hyperalgysia refers to a state of increased intensity of pain sensation induced by either noxious or ordinarily non-noxious stimulation of peripheral tissue (16), which was assessed using tail flick test, a thermal hyperalgesia test (17) and a spinal reflex, that subject to supraspinal influences $(18,19)$.

Pain complaints are commonly reported symptoms among women in mid life and later (20-23). This phenomenon suggests that ovarian hormones might play a role in modulating of pain. Neuropathic pain is one of chronic pain conditions with a predominant number of female sufferers $(24,25)$. For this reason, ovariectomised adult femelle wistar rats underwent CCI of sciatic nerve and treated with 17 beta estradiol were used to investigate the effects of neuroinflammatory processes manifested in chronic pain.

\section{MATERIAL AND METHODS}

\section{Animals}

This experimental study was performed in female adult Wister rats from the Pasteur Institute of Algiers. The rats were housed in plastic cages and were kept four weeks for adaptation at the environ- mental conditions of the experiment room (natural photoperiod, humidity, temperature etc.).

\section{Experimental design}

The animals selected with the closest possible weights and weighing approximately $217.1 \pm 6.247$ $\mathrm{g}$ were divided into four groups: Ovariectomized neuropathic untreated rats, $(n=05)$ which received subcutaneously at the dorsal region of the neck 0.1 $\mathrm{ml}$ of sesam oil for seven days. Ovariectomized neuropathic treated rats, $(\mathrm{n}=06)$ which received orally $(1,000 \mathrm{mg} / \mathrm{kg}$ of 17 beta estradiol for seven days). Sham operated $(\mathrm{n}=05)$ and intact control $(\mathrm{n}$ $=04$ ) rats.

One week before ovariectomy or sham operation, all rats were tested with Von Frey and tail flick tests successively to record the basal measurements, behavioral study was repeated after ovariectomy, after chronic constriction injury, at the first and the last day of treatment. Chronic constriction injury was recorded at the fifth week after ovariectomy. Subcutaneous injections of vehicle or treatment were carried out one week later.

\section{Surgery}

Rats were anesthetized after an intraperitoneal injection of ketamine, given a drop orally of largactil (chlorpromazin) and an ophthalmic ointment was applied to the eyes of animals using a cotton swab to avoid increased intra-ocular pressure caused by ketamine for gonadectomy, chronic constriction injury or sham surgery. The animals were placed in a calm and quiet place until fully anesthetized. Rates reflexes were checked by pinching the tip of the tail and legs with a pair of tweezers to ensure the immobility of animals before any surgery. After every operation, the animals were conscious before they were transferred to the cages where water and food were easily accessible to the animal.

\section{Depletion of ovarian hormones}

Complete ovariectomy (bilateral ovariectomy) was chosen as a common method to deplete animals of their gonadal hormones, A bilateral (left and right) incision after shaving the furs and cleansing with $70 \%$ alcohol, including skin, muscle, and peritoneum were performed $2 \mathrm{~cm}$ below the last 
rib, and the left and right ovaries were extirpated by ligation of the most proximal portion of the oviduct before removal. The muscle and the skin were subsequently sealed. The same procedure that bilateral ovariectomy was carried out for the sham groups except for the removal of the ovaries (1).

\section{Induction of peripheral neuropathic pain}

Peripheral neuropathy was induced by chronic constriction injury of left sciatic nerve where about $3-\mathrm{cm}$ long blunt dissection is made into the skin overlying the area between the gluteus and biceps femoris muscles. The common sciatic nerve of the hind paw was exposed at the mid-thigh level where approximately $7 \mathrm{~mm}$ of nerve was freed proximal to the sciatic trifurcation and four ligatures with a double knot (about $1 \mathrm{~mm}$ spacing) of 4-0 chromic guts (or 4-0 silk) were placed around the sciatic nerve until a brief twitch was observed. For sham group rats, the sciatic nerve was isolated without ligation.

\section{BEHAVIORAL MEASUREMENTS}

\section{Von Frey test}

Standard esthesiometers set which contains 20 nylon monofilaments have been used to assess mechanical sensitivity. The Up-and-Down Method was chosen to estimate mechanical paw withdrawal thresholds. The enabling principle of the Von Frey hair methodology for assessing skin sensitivity to crude touch is that a hair (or a plastic monofilament) will exert an increasing pressure on the skin as it is pressed harder and harder (26), before the filament starts to bend. But, after bending, the vertical force is constant. The force is directly proportional to the stiffness, directly related to the thickness of the filament and inversely proportional to its squared length $(27,28)$.

\section{Tail flick assay}

A lamp of $50^{\circ}$ was chosen for applying a radiant heat in direct contact with a small surface of the skin to the distal $(3.0 \mathrm{~cm}$ from the tip) of the rat's tail. The time taken for the withdrawal of the tail was taken as "tail flick" response. This latent period was considered as the index of nociception.

\section{Statical analysis}

Graphs and statistical analyses were performed using GraphPad Prism 6.01 software. The mean ( \pm standard error of the mean) values in each comparison were considered significantly different with a $\mathrm{P}$ value $<0.05$.

\section{RESULTS}

Figure1 showed a highly significant statical decrease when comparing ovariectomised neuropathic untreated group $(40.08 \pm 2.788, \mathrm{~N}=5)$ with sham operated control one $(57,42 \pm 4.217, \mathrm{~N}=5)$, (Unpaired t-test, $\mathrm{P}=0.0089)$. Fisher's exact test was reached a highly significant statical increase in comparaison of ovariectomised neuropathic treated group $(60.45 \pm 13.55 ; \mathrm{N}=6)$ with ovariectomised neuropathic untreated one $(42.40 \pm 3.114 ; \mathrm{N}=5)$, $(\mathrm{P}=0.0098)$, where no statical significat $(\mathrm{P}=$ $0.1845)$ was noted in comparaison of ovariectomised neuropathic treated group $(60.45 \pm 13.55 ; \mathrm{N}$ $=6)$ with intact control animals $(34.68 \pm 6.722 ; \mathrm{N}$ $=4$ ), (Unpaired $t$ test).

In figure 2 our data revealed an increase in anti-neutrophil cytoplasmic auto-antibodies (ANCAs) intensity plasma level after chronic constriction injury $(95.80 \pm 3.072, \mathrm{~N}=5)$ when compared with sham operated control rats $(99.20 \pm 1.855, \mathrm{~N}=$ 5), (Unpaired t test, $P=0.3712$ ). After 17beta estradiol we assessed a decrease in that plasma level $(96.40 \pm 3.326, \mathrm{~N}=5)$ in comparison with neuropathic untreated group $(98.20 \pm 5.970, \mathrm{~N}=5)$, (Unpaired t test, $\mathrm{P}=0.7989)$. The same finding was reached with statical significance $(P=0.0461$, Fisher's exact test) in comparison of intact control rats $(89.00 \pm 12.03, \mathrm{~N}=4)$ with treated ones $(96.40 \pm$ $3.326, \mathrm{~N}=5)$.

In figure 3 after chronic constriction injury we noted a reduction in the mean of ipsilateral paw withdrawal thresholds $(0.001541 \pm 0.0006616, \mathrm{~N}=$ 6), (Unpaired t test, $\mathrm{P}=0.5889$ ) when compared with sham operated control rats $(0.002284 \pm$ $0.001156, \mathrm{~N}=6$ ) where the same finding was reached for controlateral paw withdrawal thresholds $(0.003518 \pm 0.001414, \mathrm{~N}=6)$ comparing with sham operation control animals $(0.005883 \pm$ 0.003084, $\mathrm{N}=6$ ), (Unpaired t test, $\mathrm{P}=0.5015$ ).

Figure 4 showed a significant increase in the mean of ipsilateral paw withdrawal thresholds in 


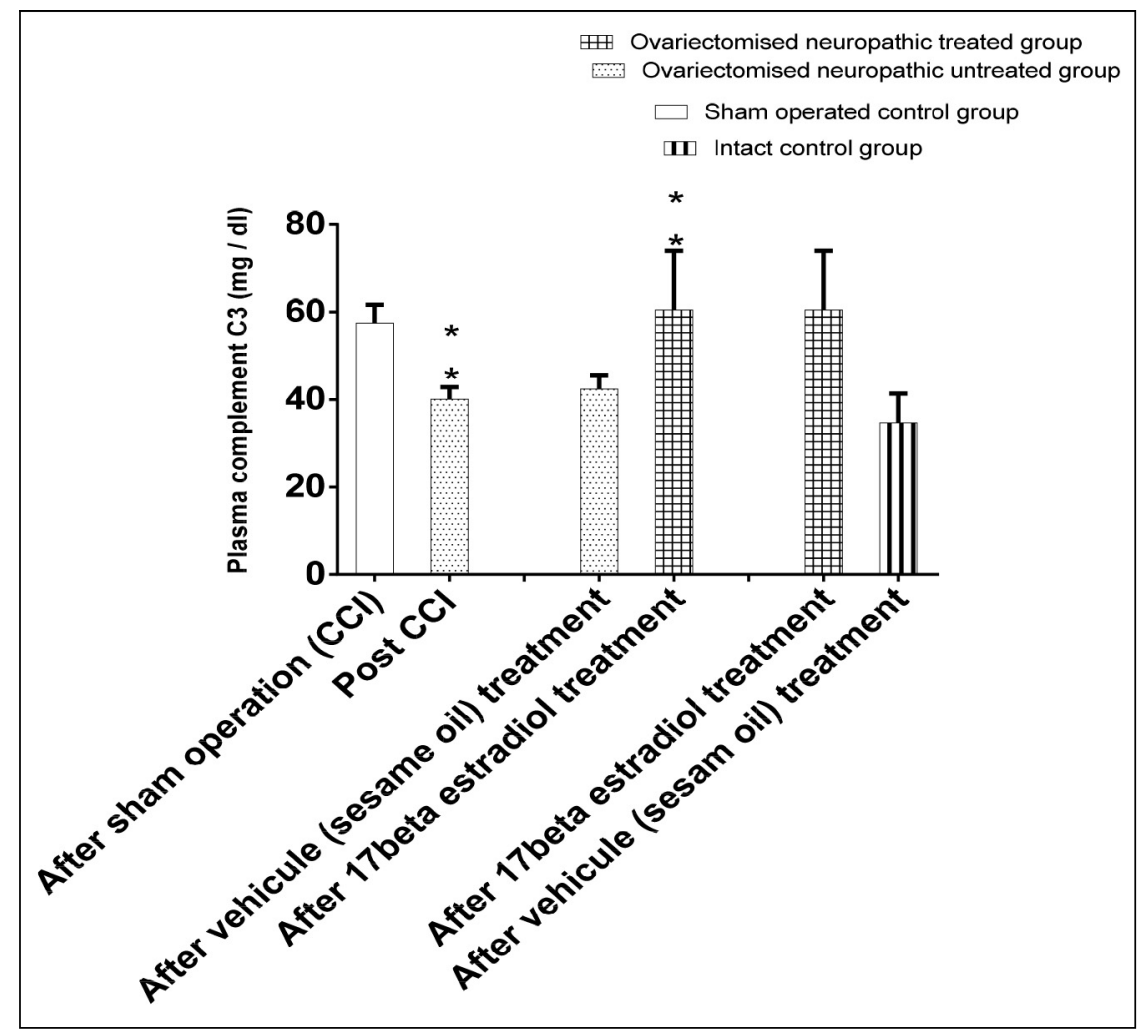

FIGURE 1. Variation of plasma level of complement C3 fractions after chronic constriction injury and after 17 beta estradiol treatment, ( $m$ $\left.\pm s ;{ }^{* *} p<0.01\right)$

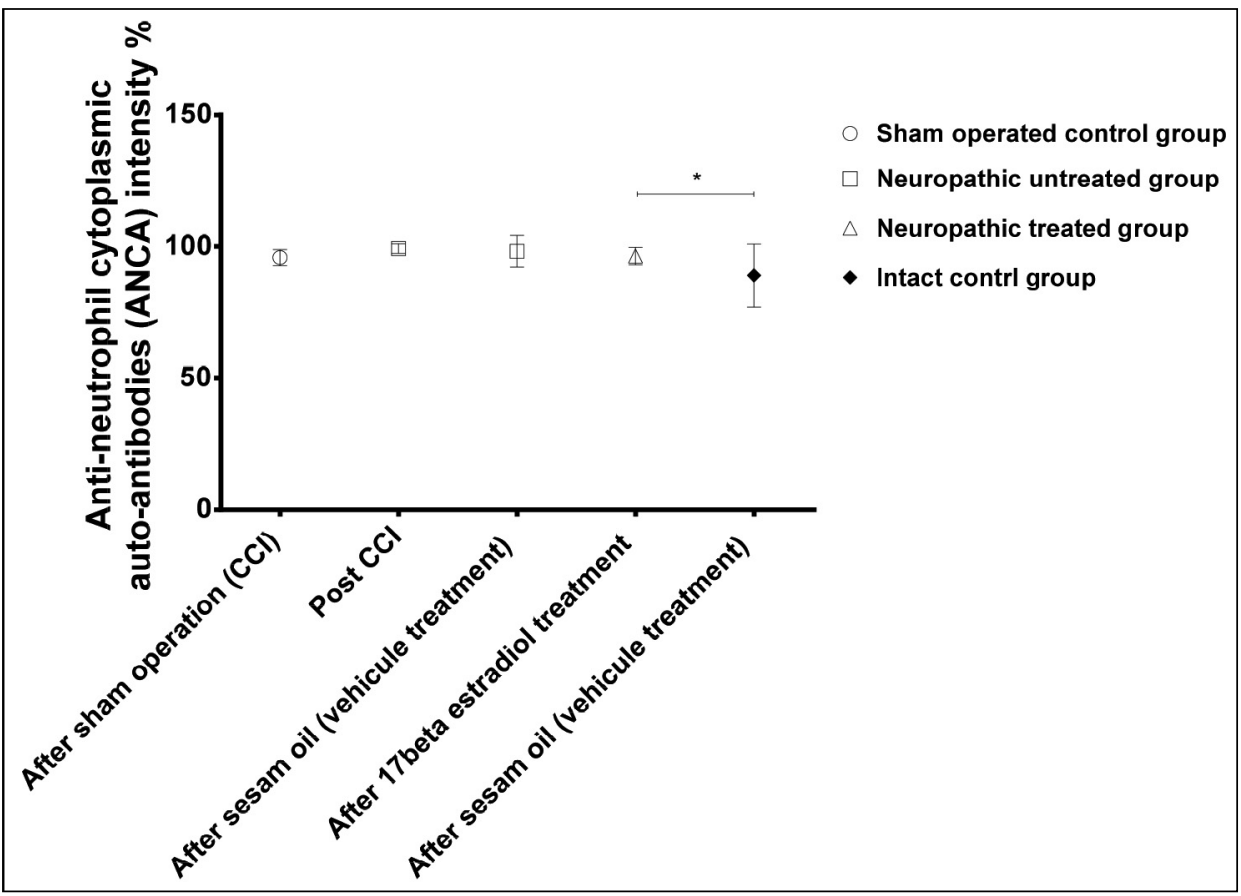

FIGURE 2. Variation of anti-neutrophil cytoplasmic auto-antibodies (ANCAs) intensity plasma level after chronic constriction injury and after 17beta estradiol treatment, $\left(m \pm s ;{ }^{*} p<0.05\right)$

17 beta estradiol-treated rats $(0.002369 \pm 0.001368$, $\mathrm{N}=6)$ when compared with untreated ones $(0.001779 \pm 0.0005020, \mathrm{~N}=6)$, (Fisher's exact test, $\mathrm{P}=0.0462)$. The values of controlateral paw withdrawal thresholds remains increased in treated animals $(0.003382 \pm 0.0008553 \mathrm{~N}=6)$ when compared with untreated ones $(0.002412 \pm 0.001004 \mathrm{~N}$ $=6$ ), (Unpaired t test, $\mathrm{P}=0.4792$ ).

Figure 5 shows that the time taken to react to heat stimulus was reduced in 17 beta estradiol treat- 

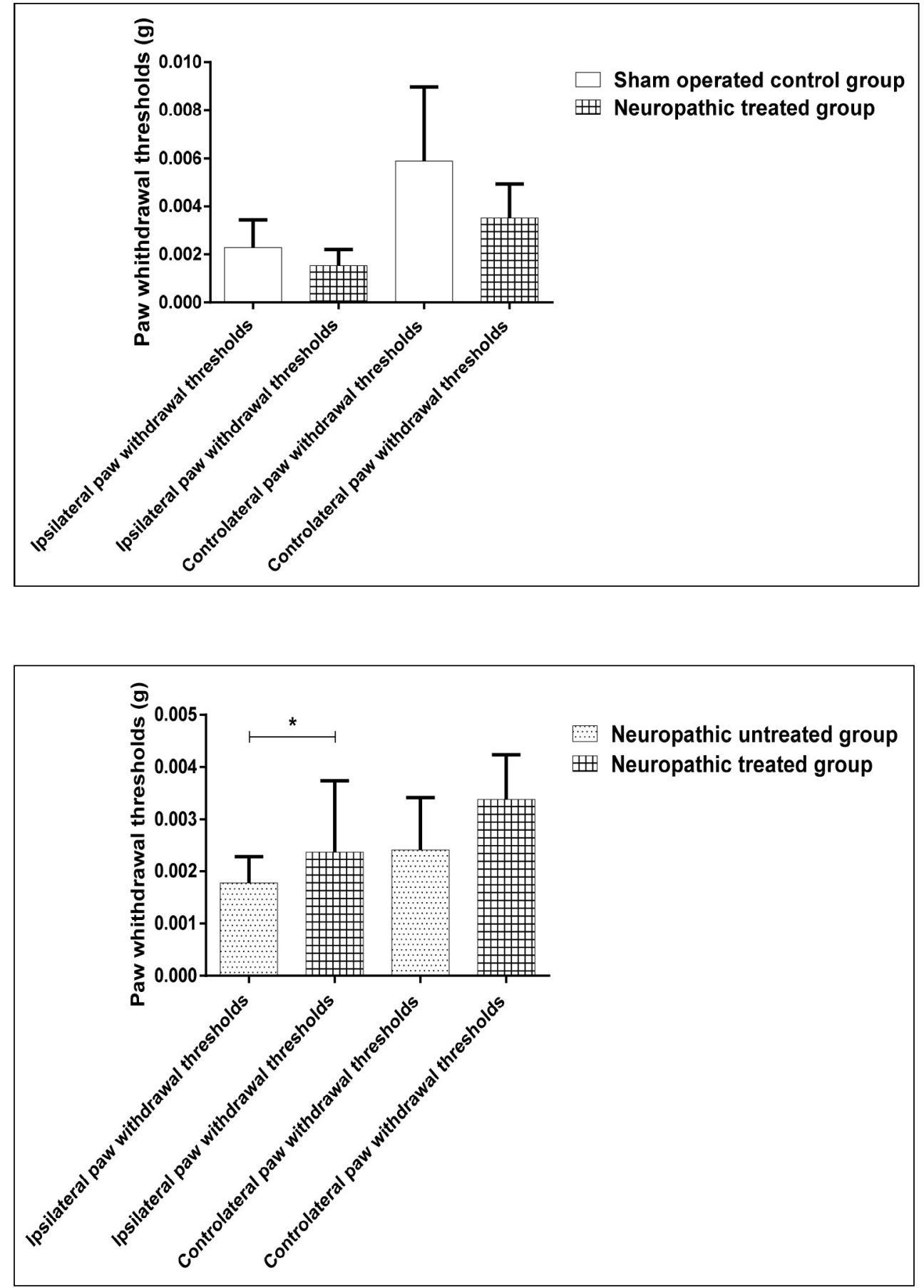

FIGURE 3. Ipsilateral and controlateral mechanical allodynia assessement after sciatic nerve chronic constriction injury
FIGURE 4. Effects of 17 beta estradiol on both hind paws withdrawal thresholds (PWT) to to mecanical stimulation ed rats after chronic constriction injury $(5.333 \pm$ $0.5577734, \mathrm{~N}=06)$ and at the first day of treatment ( $4.500 \pm 0.341565, \mathrm{~N}=06)$ in comparison with basal time $(7,667 \pm 0.9888265, \mathrm{~N}=06)$. For neuropathic untreated rats the tail withdrawal time mean was reduced after ovariectomy $(6,500 \pm 1,477611$, $\mathrm{N}=06)$ and after CCI $(3.333 \pm 0.8432741, \mathrm{~N}=06)$ in comparison with basal value $(8.167 \pm 1.327069$, $\mathrm{N}=06$ ). In contrast, at the last day of treatment in 17 beta estradiol treated rats the tail flick latency was increased $(6.667 \pm 0.95, \mathrm{~N}=06)$ than in neuropathic untreated animals $(5.333 \pm 0.9545214, \mathrm{~N}=$ 06).

\section{DISCUSSION}

The study enabled us to reveal the major consequences of peripheral nervous system damage using sciatic nerve chronic constriction injury model where all of the findings demonstrated that there is a relationship between inflammation and motor 


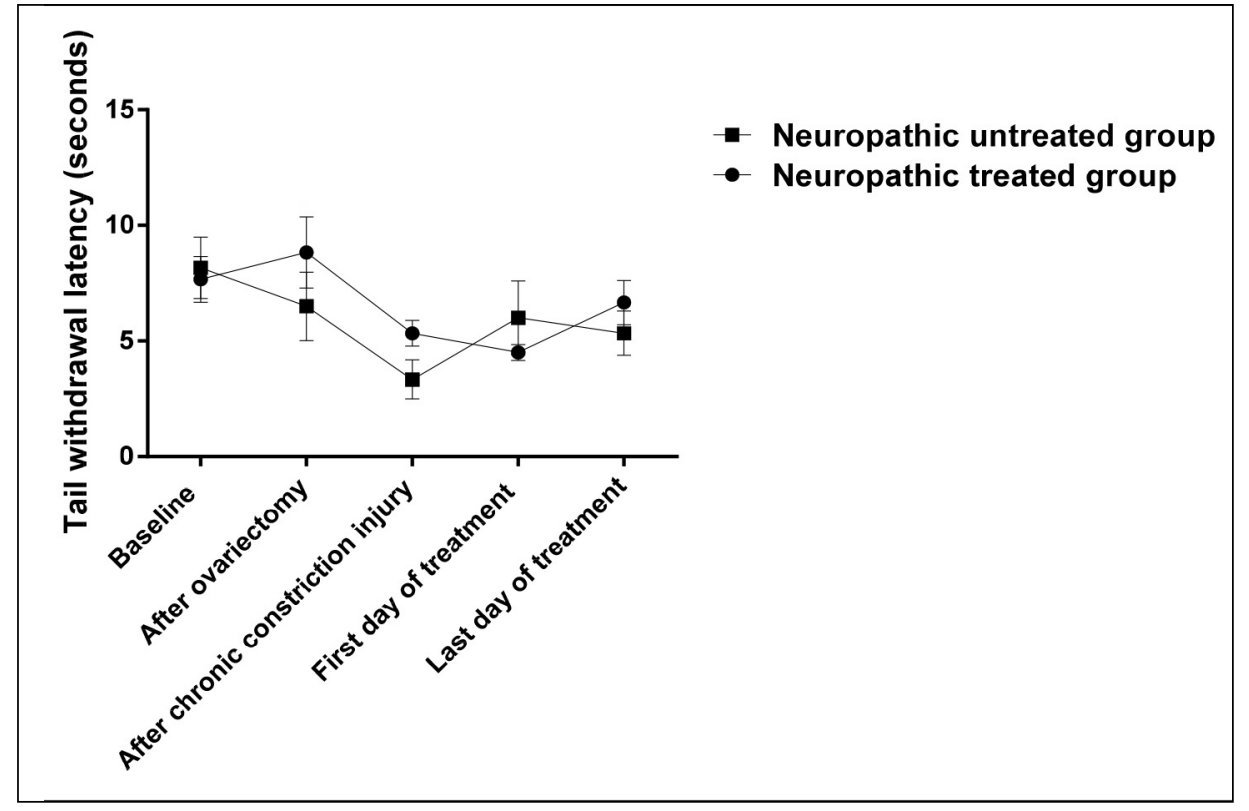

FIGURE 5. 17beta estradiol reduces thermal hyperalgesia induced after chronic constriction injury in treated rats component of pain and treatment with $17 \beta$-estradi$\mathrm{ol}$ is a modulator of those effects and exerted a neuro-protective action.

Initial peripheral nerve injuries, such as chronic constriction injury (CCI) are often compounded by secondary mechanisms, including inflammation (28). Recently, (29) demonstrated that neuroinflammation is originally serves to promote regeneration and healing after peripheral nerve injuries. The primary site of synthesis of plasma complement proteins is liver. Extra-hepatic complement biosynthesis occurs in many tissues and may account for the rapid and efficient ability of the complement system to initiate and propagate an inflammatory response (27). (28) shown endogenous synthesis and expression of components of the complement pathway in the healthy human peripheral nerve by serial analysis of gene expression (SAGE), a powerful tool of gene expression profiling. mRNAs encoding activating, inhibitory and regulatory components of the complement system were highly represented in the SAGE and their expression was confirmed by northern blot and RTPCR. Epithelial cells (30), fibroblasts (31), Schwann cells (32) and macrophages (33) can synthesize complement in vitro but the high expression level, determined by SAGE, supports the hypothesis than Schwann cells are likely the main source of complement mRNA in the peripheral nerve trunk. Moreover, (34) demonstrated that C3 activate as- trocytes and induce inflammatory signaling (TNF- $\alpha$ ) and concluded that C3 and other complement proteins are likely participate in non-traditional roles as mediators of axon regeneration and neuronal survival/adhesion. Our result showed a reduction in complement plasma level after nerve injury which indicates that $\mathrm{C} 3$ is consumed, for the reason that a majority of the $\mathrm{C} 3$ protein in the human serum or plasma is in the native (or intact) state, however, when $\mathrm{C} 3$ is activated (e.g., disease, infection, or tissue injury), the native protein is processed into various proteolytic cleavage products ('split products') including $\mathrm{C} 3 \mathrm{a}, \mathrm{iC} 3 \mathrm{~b}, \mathrm{C} 3 \mathrm{c}, \mathrm{C} 3 \mathrm{~d}$, etc (35). Our finding was supported and augmented by murings of studies, (28) demonstrated that transection or crush injury to peripheral axons results in rapid activation (within 1 hour) of the complement cascade locally at the site of injury and as a retrograde response, in the motor nuclei and sensory projections laying in the CNS demonstrated (3640). In their study on spinal cord injury (SCI), C1q, factor $\mathrm{B}$ and $\mathrm{C} 3$ are increased in sciatic nerves of humans with traumatic nerve lesion-induced pain and after spinal cord injury in rodents. In SCI model, the complement cascade has been shown to be activated after peripheral nerve injury and to play a role in hypersensitivity through the release of the anaphylatoxin peptide (C5a) by the terminal membrane attack complex (MAC) complement, itself induced by $\mathrm{C} 3$ activity. In their study carried out on 
sural nerve biopsy samples taken from patients with nonsystemic vasculitic neuropathy (NSVN) and microscopic polyangiitis (MPA), complement component $\mathrm{C} 3 \mathrm{~d}$ deposits around epineurial vessels. Although epineurial vessels that exhibited signs of vasculitis, such as destruction of vessel walls accompanied by inflammatory cellular infiltration, showed some positive anti-C3d antibody staining, some epineurial vessels with preserved morphology also stained positively. When these vessels were assessed with preserved morphology, C3d deposition was more frequently observed in patients with NSVN than in those with microscopic polyangiitis-associated neuropathy (MPAN). Pain behavior was significantly attenuated in SNL rats treated with CVF as was complement activity at the ipsilateral dorsal root ganglia which suggest that the complement pathway might be a novel target for the development of neuropathic pain therapeutics. In a modified classical chronic constriction injury (mCCI) model, once complement is activated, cascade reaction occurs rapidly leading to producing end products of terminal complement C5b-9 complex, i.e. C5b-8 complex and MACs, making a pinhole in target cell membrane. Moreover, C5a-induced thermal hyperalgesia. Their findings suggest that C5a generated in response to injury or inflammation acts via macrophages to initiate an intercellular signaling cascade that ultimately causes sensitization of nociceptors to heat. Estrogens influence immune processes, where their effects on metabolic improvement may be a consequence of regulation of inflammation pathways. After 17-beta estradiol treatment, we assessed an increase in complement plasma level, because of complement component $\mathrm{C} 3$ plays a central role in the activation of complement system. Its activation is required for both classical and alternative complement activation pathways. The encoded preproprotein is proteolytically processed to generate alpha and beta subunits that form the mature protein, which is then further processed to generate numerous peptide products. The $\mathrm{C} 3 \mathrm{a}$ peptide, also known as the $\mathrm{C} 3 \mathrm{a}$ anaphylatoxin, modulates inflammation and possesses antimicrobial activity (10). Estrogen induces synthesis of complement $\mathrm{C} 3$ in the epithelium of the immature rat uterus. Moreover, the uterus of the immature rat synthesizes and secretes complement component $\mathrm{C} 3$ in response to estradiol treatment.
This response occurs in the uterine epithelial cells. Uterine luminal epithelial cells synthesized and secreted $\mathrm{C} 3$ only after $\mathrm{E}_{2}$ administration for 3 days in ovariectomised female rats, whereas the uteri from control animals did not produce $\mathrm{C} 3$. In contrast, the ectopic endometrium from control animals produced and secreted $\mathrm{C} 3$, and this expression was strongly upregulated by in vivo $\mathrm{E}_{2}$ administration. Sciatic nerve regeneration involves several overlapping stages, including apoptosis and inflammation, Schwann cell activation/proliferation, and myelin remodeling and confirmed that steroid hormones, in particular $17 \beta$-estradiol, control reactive gliosis modifying the number of reactive astrocytes and reactive microglia and the expression of proinflammatory mediators in sciatic nerve ligation model. Expression of C1q subunit B (C1Q), an initiating factor of the classical complement pathway, was higher in males and was suppressed in both sexes in response to EE2 and bacterial challenge. Moreover, cleavage and post-translational modification of $\mathrm{C} 3$, the central component of the complement could be altered by EE2 treatment in males (C3dg down; C3g up). In their study carried out on sciatic nerve ligation, it has been correlated to a higher neuronal survival and also to a functional improvement, since the neuroglobin has antioxidant as well as anti-apoptotic and anti-inflammatory properties. The regulation of neuroglobin expression has been identified as one of the molecular mechanisms that mediates estradiol in neuroinflammation, in particular its anti-inflammatory properties. Inflammation is the process by which an organism responds to tissue injury involving immune cell recruitment. The first cells to react to damage of the nerve are schwann cells and resident immune cells such as mast cells and macrophages where resident mast cells degranulate releasing inflammatory mediators which can sensitize nociceptors and also contribute to the recruitment of neutrophils, the first cells to infiltrate damaged tissue. Neutrophils have been shown to invade the ipsilateral dorsal root ganglion (DRG) after peripheral nerve injury between 7 and 14 days post-surgery (depending on the injury) much later than that observed peripherally, and also at lower levels, ANCAs (Anti-neutrophil cytoplasmic auto-antibodies) are a group of auto-antibodies directed against cytoplasmic antigens localized in neutrophils (the 
most common type of white blood cell), where serum levels of ANCA are used in the purposes of diagnosis, prognosis and in monitoring of inflammatory activity. Our data revealed an increase in ANCA intensity after nerve injury where the finding was confirmed by a diversity of studies. A case of a 19-year-old woman with chronic inflammatory demyelinating polyneuropathy (CIDP) with high PR3-ANCA positivity where they suggested that CIDP may accompany high PR3-ANCA levels, which should be differentiated from axonal neuropathy due to vasculitis. aß2GP I and p-ANCA levels may imply the danger of the occurrence of neuropathy in (Sjogren's syndrome) SJS patients, and they can be considered a biomarker that should be added to the panel of conventional autoantibody in SJS patients. Positive anti-Ro antibody may imply neuropathy in Lupus nephritis (LN) patients and can be considered a biomarker that should be added to the panel of conventional autoantibodies in LN patients. After 17beta estradiol we revealed a reduction in ANCA intensity, our finding was supported by murings of studies. The patient with chronic inflammatory demyelinating polyneuropathy (CIDP) responded well to intravenous immunoglobulin plus oral steroid. The levels of neutrophil serine proteases (NSPs) and myeloperoxidase (MPO) were highly upregulated in the splenocytes from estrogen-treated mice. Peripheral nerve injuries and diseases often lead to pain persisting beyond the resolution of damage, indicating an active disease-promoting process, which may result in chronic pain. This is regarded as a maladaptive mechanism resulting from neuroinflammation. Neuropathic pain manifests with a range of different symptoms such as ongoing burning pain, squeezing or pressure pain, paroxysmal electric shock-like sensations, stabbing pain, or mechanical dynamic allodynia and is characterized by hyperalgesia. Our resuts showed a reduction in both ipsilateral and controlateral paw withdrawal thresholds after chronic constriction injury when compared with those in sham operated rats which reveal a development of ipsilateral and controlateral allodynia in CCI animals. The finding corroborated with numerous of studies. Mechanical allodynia and functional recovery of the injured paw in sciatic nerve chronic constriction injury (CCI) model was followed for 101 days. In carrageenan-induced mir- ror-image pain demonstrated that the expression of ipsilateral D-serine was up-regulated during the early phase of inflammation, while contralateral D-serine increased during the later phase of inflammation. In carrageenan-induced mirror-image pain demonstrated that the expression of ipsilateral D-serine was up-regulated during the early phase of inflammation, while contralateral D-serine increased during the later phase of inflammation. In our study, 17beta estradiol exerted an ipsilateral and controlateral anti-allodynic actions, this finding extended with other results, (31) investigated that the hippocampal TRPV1 (transient receptor potential vanilloid 1) expression in ovariectomized rats that received 17- $\beta$-estradiol substitution and found that $17-\beta$-estradiol enhanced the mechanical allodynia of inflamed temporomandibular joint (TMJ) induced by complete Freund's adjuvant. In addition, Real-time PCR and immunoblotting demonstrated that TMJ inflammation significantly induced hippocampal TRPV1 expression compared with the control group but failed to induce it in the ovariectomized rats that received no estradiol replacement. Moreover, estradiol potentiated TMJ inflammation-induced hippocampal TRPV1 expression in a dose-dependent manner in the ovariectomized rats. (31) demonstrated that Male mice show a gradual decrease of allodynia and a complete recovery while, in females, allodynia and gliosis were still present four months after neuropathy induction. Administration of $17 \beta$-estradiol was able to significantly attenuate that difference, reducing allodynia and inducing a complete recovery also in female mice. Parallel to pain attenuation, 17 $\beta$-estradiol treated-mice showed a functional improvement of the injured limb, a faster regenerative process of the peripheral nerve and a decreased neuropathy-induced gliosis. Their results indicated beneficial effects of $17 \beta$-estradiol on neuropathic pain and neuronal regeneration and focuses on the importance of considering gonadal hormones also in clinical studies. In Fibromyalgia (FM): a musculoskeletal chronic pain syndrome, the absence of ovarian hormones (in OVX rats) increased muscle nociception. 17 $\beta$-estradiol produced anti-hyperalgesic and anti-allodynic effects $24 \mathrm{~h}$, but not $8 \mathrm{~h}$, after its administration, suggesting a genomic mechanism. The results supported the validity of the reserpine-induced FM model 
for searching alternatives of treatment, particularly during endocrine phases when pain is exacerbated such as menopause, and that $17 \beta$-estradiol replacement might be useful.

In hyperalgesia assessment and using tail flick test, one of the commonly accepted models for quantifying analgesic response in animals. Our data revealed a hyperalgesia development after ovariectomy and chronic constriction injury in neurpopathic untreated rats where the same finding was reached after CCI and at the first day of treatment in treated animals, the outcome led after 17 beta estradiol administration was attenuating hyperalgesia by increasing the tail flick latency in comparison with neuropathic (sesame oil) vehicule rats. OVX mice, developed mechanical hyperalgesia localized to the abdominal region, the hind limbs and at the proximal tail in a model of functional abdominal pain where the estrogen reversed both mechanical and thermal hyperalgesia. Estradiol could modulate trigeminal ganglionic Nav1.7 expression to enhance hyperalgesia of inflamed temporomandibular joint (TMJ). Moreover, estradiol is anti-hyperalgesic after carrageenan administration by increaseing paw withdrawal latencies (PWL) to a thermal stimulus.

Sciatic nerve chosed sciatic nerve chronic ligation was chosen as a chronic pain model that made a significant contribution in understanding the pathophysiological mechanisms in chronic pain, which is quite distinct from acute noxious pain. One of the most commonly employed animal model of neuropathic pain. The model produces unilateral peripheral mononeuropathy, and it has been observed that symptoms in this rat model correspond to causalgia or complex regional pain syndrome in patients. It induces allodynia and hyperalgesia in rodents and other symptoms which are similar to those of neuropathic pain in humans.
In terms of limitations accompanying diseases, malingering and placebo effects were not be taken.

More work is needed for determination of the most predictive animal models, removal of user bias and introduction of more complex outcome measures in behavioral tests. It is important to state that in pain research the problem is even more pronounced due to the subjective nature of painful experience. Only humans have the ability to express and describe the emotional aspect of a painful experience.

\section{CONCLUSIONS}

In conclusion, chronic constriction injury (CCI) animal model is more suitable for the long-term observation and research of peripheral nerve injury-induced neuropathic peripheral pain (NPP). In this study, CCI induced a significant increase in expression of complement C3, ANCAs (Anti-neutrophil cytoplasmic auto-antibodies) and was consistent with the occurrence of hypersensivity. Administration of 17 beta estradiol reversed the inflammatory reactions involved in the formation of hypersensivity induced by CCI.

\section{Compliance with ethical statements}

The experimental protocol was approved by the Scientific Committee of our faculty which is consistent with the principles of Animal Health (NIH Publication No. 85-23, revised 1985). All applicable international, national, and/or institutional guidelines for the care and use of animals were followed.

\section{Acknowledgement}

This work is done within the framework of national research project Code D01N01UN2 30120150001 in Algeria (CNEPRU).

Conflict of interest: none declared

\section{REFERENCES}

1. Steve K Lee, MD, Scott W. Wolfe, MD. Peripheral Nerve Injury and Repair. Journal of the American Academy of Orthopaedic Surgeons. 2000 Jul-Aug; 8(4):243-252.

2. Scollard DM. The biology of nerve injury in leprosy. Lepr Rev. 2008 Sep;79(3):242-253.

3. Birdi TJ, Antia NH. Mechanisms involved in peripheral nerve damage in leprosy with special reference to insights obtained from in vitrostudies and the experimental mouse model. Int $\mathrm{J}$ Lepr Other Mycobact Dis. 2003 Dec;71(4):345-354.
4. Scollard DM, Truman RW, Ebenezer GJ. Mechanisms of nerve injury in leprosy. Clin Dermatol. 2015 Jan-Feb;33(1):46-54.

5. Ellis $A$, Bennet DLH. Neuroinflammation and the generation of neuropathic pain. British journal of Anaesthesia. 2013 Jul;111(1):26-37.

6. Perkins NM, Tracey DJ. Hyperalgesia due to nerve injury: role of neutrophils. Neuroscience. 2000;101(3):745-757.

7. Zuo Y, Perkins NM, Tracey DJ, Geczy CL. Inflammation and hyperalgesia induced by nerve injury in the rat: A key role of mast cells. Pain. 2003 Oct; 105(3):467-79. 
8. Fleming JC, Norenberg MD, Ramsay DA, Dekaban GA, Marcillo AE, Saenz AD, Pasquale-Styles M, Dietrich WD, Weaver LC. The cellular inflammatory response in human spinal cords after injury. Brain. 2006 Oct 28;129(Pt 12):3249-3269.

9. DiSabato D, Quan N, Godbout J P. Neuroinflammation: The Devil is in the Details. J. Neurochem. 2016 Oct;139 Suppl 2:136-153.

10. Fachuan Niel, Jinbao Wang, Dong Su, Ying Shi, Jinmei Chen, Haihui Wang, Wanxiang Qin, Lin Shi. Abnormal activation of complement C3 in the spinal dorsal horn is closely associated with progression of neuropathic pain. International Journal of Molecular Medicine. 2013 Apr; 31(6)1333-1342.

11. Davoust N, Jones J, Stahel PF, Ames RS, Barnum SR. Receptor for the C3a anaphylatoxin is expressed by neurons and glial cells. Glia. 1999 May;26(3):201-211.

12. Dworkin RH, Backonja M, Rowbotham MC, Allen RR, Argoff CR, Bennet GJ, Bushnell MC, Farrar JT, Galar BS, Haythornthwaite JA, Hawitt DJ, Loeser JD, Max MB, Saltarelli M, Schmader KE, Stein C, Thompson D, Turk DC, Wallace MS, Watkins LR, and Weinstein SM. Advances in neuropathic pain: Diagnosis, mechanisms, and treatment recommendations. Arch Neurol. 2003;60(11):1524-1534.

13. Hama AT, Borsook D. Behavioral and pharmacological characterization of a distal periheral nerve injury in the rat. Pharmacol Biochem Behav. 2005 May;81(1):170-181.

14. Jurgen Sandkuhler. Models and Mechanisms of Hyperalgesia and Allodynia. Physiol.Rev. 2009 Apr;89(2):707-58.

15. Abelson Klas, Roughan, John V. Animal Models in Pain Research. In: Jann Hau, Steven J Schapiro. (eds.) Handbook of Laboratory Animal. Boca Raton/ New York: CRC Press; 2011:129.

16. Hardy JD, Wolff HG, Goodell H. Experimental evidence on the nature of cutaneous hyperalgesia. J Clin Invest. 1950 Jan;29(1):115-140.

17. Neurofit. 2017. Preclinical Research for CNS and PNS disorders. http://www. neurofit.com/tech-anim-tail-flick.html. Accessed 2018.

18. Yaksh TL, Rudy TA. Narcotic analgestics: CNS sites and mechanisms of action as revealed by intracerebral injection techniques. Pain. 1978 Apr;4(4):299-359.

19. Millan MJ. Descending control of pain. Prog Neurobiol. 2002 Apr; 66(6):355-474.

20. Brown WJ, Mishra GD, Dobson A. Changes in physical symptoms during the menopause transition. Int J Behav Med. 2002;9(1):53-67.

21. Dugan SA, Powell LH, Kravitz HM, Everson Rose SA, Karavolos K, Luborsky J. Musculoskeletal pain and menopausal status. Clin J Pain. 2006 May;22(4):325-331.

22. Freeman EW, Sammel MD, Lin H, Gracia CR, Kapoor S. Symptoms in the menopausal transition: hormone and behavioral correlates. Obstet Gynecol. 2008 Jan;111(1):127-36.

23. Freeman EW, Sammel MD, Lin H, Gracia CR, Pien GW, Nelson DB, Sheng L. Symptoms associated with menopausal transition and reproductive hormones in midlife women. Obstet Gynecol. 2007 Aug; 110(2 Pt 1):230-40.

24. Riley JL, Robinson ME, Wise EA, Myers, CD, Fillingim RB. Sex differences in the perception of noxious experimental stimuli: a meta-analysis. Pain. 1998 Feb;74(2-3):181-7.
25. Fillingim RB, King CD, Ribeiro-Dasilva MC, Rahim-Williams B, Riley JL. Sex, gender, and pain: a review of recent clinical and experimental findings. J Pain. 2009 May;10(5):447-85.

26. Wood Dale, 620 Wheat Lane, Wood Dale, IL 60191. 2001. Touch Test $^{\mathrm{TM}}$ Sensory Evaluators Semmes Weinstein Von Frey Aesthesiometers. United States of America, Stoelting Co, 2001.

27. Fruhstorfer $\mathrm{H}$, Gross W, Selbmann $\mathrm{O}$. Von Frey hairs: new materials for a new design. European Journal of Pain. 2001;5(3):341-342.

28. Mogil JS, Wilson SG, Wan Y. 2001. Assessing nociception in murine subjects. In Methods in pain research, ed Kruger L, 11-39. Boca Raton: CRC Press.

29. Azizi-Malekabadi H, Hosseini M, Soukhtanloo M, Sadeghian R, Fereidoni M, Khodabandehloo F. Different effects of scopolamine on learning, memory, and nitric oxide metabolite levels in hippocampal tissues of ovariectomized and sham-operated rats. Arquivos de Neuro-Psiquiatria. 2012 Jun;70(6):447-452.

30. Hossein Ali Safakhah, Nasroallah Moradi Kor, Atiyeh Bazargani, Ahmad Reza Bandegi, Hamid Gholami Pourbadie, Baharak Khoshkholgh-Sima, Ali Ghanbari. Forced exercise attenuates neuropathic pain in chronic constriction injury of male rat: an investigation of oxidative stress and inflammation. Journal of Pain Research. 2017 June;2017(10):1457-1466.

31. Sommer C, Leinders $M$, Üçeyler N. Inflammation in the pathophysiology of neuropathic pain. J pain. 2018 Mar;159(3):595602.

32. Ramaglia V, Daha M, and Baas F. The complement system in the peripheral nerve: friend or foe? Molecular Immunology. 2008 Sep; 45(15):3865-3877. Review.

33. de Jonge RR, van Schaik IN, Vreijling JP, Troost D, Baas F. Expression of complement components in the peripheral nervous system. Hum Mol Genet. 2004 Feb 1;13 (3):295-302.

34. Strunk RC, Eidlen DM, Mason RJ. Pulmonary alveolar type II epithelial cells synthesize and secrete proteins of the classical and alternative complement pathways. J Clin Invest. 1988 May; 81(5):1419-1426.

35. Katz Y, Strunk RC. Synthesis and regulation of complement protein factor H in human skin fibroblasts. J Immunol. 1988 Jul 15; 141(2):559-563.

36. Dashiell SM, Vanguri P, Koski CL. Dibutyryl cyclic AMP and inflammatory cytokines mediate C3 expression in Schwann cells. Glia. 1997 Aug 1; 20(4):308-321.

37. McPhaden AR, Whaley K. Complement biosynthesis by mononuclear phagocytes. Immunol Res. 1993; 12:213-232.

38. Sheri L Peterson, Hal X Nguyen, Oscar A, Mendez, Aileen J, Anderson. Complement Protein C3 Suppresses Axon Growth and Promotes Neuron Loss. Scientific Reports. 2017 Oct 10;7

39. Liu HM, Yang LH, Yang YJ. Schwann cell properties: 3. C-fos expression, bFGF production, phagocytosis and proliferation during Wallerian degeneration. J Neuropathol Exp Neurol. $1995 \mathrm{Jul}$; 54(4):487-496. 